Revue d'histoire de l'Amérique française

REYUE D.HISTOIRE DE L'AMÉRIQUE FRANÇAISE

\title{
RAPPORT PARENT - rapport de la Commission royale d'enquête sur l'enseignement dans la province de Québec. Imprimé et relié par Ronalds-Federated Limited, Imprimeur-relieur, pour le Gouvernement du Québec. 1966.
}

\section{Lionel Groulx}

Volume 20, numéro 3, décembre 1966

URI : https://id.erudit.org/iderudit/302595ar

DOI : https://doi.org/10.7202/302595ar

Aller au sommaire du numéro

Éditeur(s)

Institut d'histoire de l'Amérique française

ISSN

0035-2357 (imprimé)

1492-1383 (numérique)

Découvrir la revue

Citer ce compte rendu

Groulx, L. (1966). Compte rendu de [RAPPORT PARENT - rapport de la Commission royale d'enquête sur l'enseignement dans la province de Québec. Imprimé et relié par Ronalds-Federated Limited, Imprimeur-relieur, pour le Gouvernement du Québec. 1966.] Revue d'histoire de l'Amérique française, 20(3), 458-466. https://doi.org/10.7202/302595ar d'utilisation que vous pouvez consulter en ligne. 
RAPPORT PARENT - rapport de la Commission royale d'enquête sur l'enseignement dans la province de Québec. Première partie ou tome I: Les structures supérieures du système scolaire; Deuxième partie ou tome II: Les structures pédagogiques du système scolaire - A - Les structures et les niveaux de l'enseignement; Deuxième partie ou tome II (suite) : Les structures pédagogiques du système scolaire A -Les programmes d'études et les services éducatifs; Troisième partie: L'administration de l'enseignement - A - Diversité religieuse, culturelle, et unité de l'administration; Troisième partie ou tome III (suite): L'administration de l'enseignement - B - Le financement; C Les agents de l'éducation. Imprimé et relié par RonaldsFederated Limited, Imprimeur-relieur, pour le Gouvernement du Québec. 1966.

Les Editions Fides offrent au public, à titre de "distributeur exclusif", le Rapport Parent, en cinq petits volumes de poche. L'un de ces volumes, le deuxième, dépasse les 400 pages, d'autres atteignent à ce même nombre ou peu s'en faut; le premier et le quatrième restent beaucoup en deçà. Somme toute: 1469 pages. L'ouvrage n'en est pas moins de consultation facile. 
Et comme les répercussions du document dans la vie canadiennefrançaise peuvent paraître indéfinies et indéfinissables, il importait qu'il fût mis, sous forme commode, à la portée du public. On peut regretter toutefois que le dernier volume ne contienne pas des tables générales: table onomastique et table des matières qui permettraient de se retrouver dans l'immense ouvrage. Mais, de toute façon, le Rapport est devenu un document historique et par soi-même et par l'usage que l'on en a fait. Et c'est à ce titre qu'il en est question ici.

Il y a d'excellentes choses dans le Rapport. La mise à portée de l'enseignement, de quelque forme que ce soit, à tout enfant de la nation qui s'en révèle capable, ne peut souffrir aucune critique. L'école polyvalerite, pourvu qu'on en fixe les options à un âge raisonnable, et qu'on soit en état d'y fournir les locaux, les laboratoires, les bibliothèques, et surtout la légion de maîtres compétents qu'il y faudra, n'est pas, non plus, de nature discutable. L'introduction d'une étude plus poussée de toutes les sciences s'impose de première nécessité. Nous en dirons autant de la formation à la recherche: excellent apprentissage de l'esprit à la curiosité, au souci de l'exactitude. Encore serait-il bon que le maître connût lui-même les sujets de travaux qu'il propose à ses élèves et que, pour leurs recherches, il pût fournir la bibliothèque où s'informer, ou du moins, où s'adresser. Nous en savons quelque chose, nous, entre autres, à notre Institut où nous recevons, avec grand plaisir, du reste, collégiens et collégiennes à qui l'on propose des travaux bien au-dessus de leur capacité d'esprit et sans leur fournir le moindre bout de bibliographie. Egalement beaucoup à louer l'éducation permanente ou ce que l'on appelle le "recyclage" de la main-d'œuvre ouvrière. En 1941, nous dénoncions nous-mêmes, dans l'Action nationale, la honteuse proportion de nos ouvriers non-qualifiés qui nous plaçait au-dessous de la plupart des Néo-Canadiens. A l'unité de direction et à la coordination dess divers enseignements dans le respect des libertés essentielles, personne ne trouvera à redire. D'ailleurs, en son propos, l'auteur de ce compte rendu n'entend pas s'ériger en panégyriste, non plus qu'en dénigreur systématique du Rapport. Sa position se peut définir très nette. Il ne se pique nullement d'être un catholique "dans le vent", depuis que tant d'idées échevelées courent le monde. Il lui suffirait de se savoir un catholique de foi toute simple, de la foi que l'Eglise enseigne et maintient même après le Concile.

L'avouerai-je, en outre ? J'étais de ceux qui souhaitaient vivement une réforme de notre enseignement et surtout de notre 
éducation humaine, nationale et chrétienne. Rien ne me serait plus facile que d'apporter ici des textes où j'ai déploré, en notre enseignement primaire, son affreux vide, son manque trop fréquent d'ouverture, surtout dans les villes où l'on éduquait les fils d'un petit peuple sans le moindre souci du terrible milieu où il avait à vivre sa vie. J'ai regretté, et tout autant, l'anémique formation religieuse, le pauvre enseignement des humanités, de la philosophie, dans nos institutions d'enseignement secondaire. Enseignement trop artificiel, trop étranger, lui aussi, à son milieu. Je n'ai pas non plus ménagé mes critiques à nos universités, y apercevant si peu de l'esprit qui eût dû être celui de ces hautes institutions, incapables de prendre les devants dans les réformes qui ne pouvaient plus attendre et n'étant rien moins, pour la nation, que des écoles de chefs.

Ceci dit, nous exposerons franchement nos critiques sur le Rapport. Nous estimons malheureux que les commissaires aient bâti leur système d'enseignement en marge de l'ancien, sinon même tout à fait à côté. Tout n'était pas vermoulu, inadapté dans l'ancien système que, depuis deux cents ans tout près, dans la misère et la pure charité, avait édifié le peuple canadienfrançais. Si grevé de lacunes qu'on ait voulu le dire, il n'y avait pas là qu'accumulation de sottises. Ce vieux système, on s'était efforcé de l'ajuster à nos traditions, à ce que l'on appelle l'âme d'une nation, d'un pays. Et par son indéniable rajeunissement déjà mené à vive allure, par ses édifices, ses bibliothèques, ses laboratoires mis à point, et encore par son personnel d'enseignants de plus en plus qualifiés, il y avait là un actif, m'ont dit les économistes, qu'on eût pu évaluer à des milliards. Quelle nécessité y avait-il de renvoyer tout cela au vieux fer, comme l'on eût fait d'un vaisseau hors d'usage ? Ne s'y trouvait-il vraiment nulle pièce qui, réformée, remodelée si l'on veut, n'eût pu entrer dans le nouvel édifice, surtout quand l'on avait tant besoin de locaux, de maîtres et de tout l'équipement scolaire?

D'où venait, au surplus, et ici nous ne mettons pas en cause les commissaires, d'où venait cette hâte de conférer au Rapport une sorte de valeur législative, quand ce Rapport, comme tous les rapports de même nature, ne revêtait d'autre importance ni d'autre qualité qu'une recommandation au gouvernement? Dès la publication de ses premières tranches, le document devint tabou, aussi sacré que certain décalogue descendu du Sinaï. De ce fétichisme se firent adeptes malheureusement tous les médiums de publicité. On eût dit un mot d'ordre réprouvant toute critique. Je ne cite qu'un exemple: Relations, revue des Jésuites de Mont- 
réal, publia une livraison spéciale tirée à plus de 30,000 exemplaires. Des critiques parfois sévères s'y trouvaient; le plus souvent des articles d'un ton modéré, et voire, au milieu de mises en garde fort opportunes, quelques articles approbateurs. Or la "livraison spéciale" passa presque inaperçue. Ce silence avec bien d'autres furent un malheur, et pour les commissaires qui allaient se permettre d'autres témérités, et pour les empressés metteurs en œuvre du Rapport qui, pour donner efficacité et vie à leur système, se verraient forcés à ce que nos gens appellent du "raboudinage": rajuster ceci et cela, ressaisir ce qu'ils avaient trop tôt rejeté, en particulier les écoles normales. Il fallait aller vite, paraît-il, au mépris de l'axiome qui veut que s'il est bon d'aller vite, il est rarement bon d'aller trop vite.

Où j'ai quelque peine également à donner raison aux Commissaires, c'est dans leur volontaire ou involontaire obscurité au sujet de la confessionnalité scolaire. Doctrine confuse à tout le moins. Le divorce est évident entre enseignement et éducation. On ne parle plus que d'enseignement religieux. On devrait savoir pourtant qu'en l'école confessionnelle, l'élément, l'esprit religieux est l'âme vivante et qu'il y faut non seulement enseigner, mais former. Et quelle idée avissi que de soumettre l'exercice de la confessionnalité à des organismes neutres ! Oh ! je sais derrière quelles excuses ils se pourraient retrancher. Combien est confuse elle-même la pensée des catholiques québecois sur ce sujet. Combien de clercs dits d'avant-garde, combien de commissaires d'écoles catholiques, pris soudain de je ne sais quelle incommensurable humilité ou de quel œuménisme plus que discutable, seraient prêts à tout immoler sur l'autel du pluralisme religieux; ils réduisent la religion de leur province de l'état majoritaire à l'état minoritaire et d" "une minorité comme les autres"; ils optent par conséquent pour la confessionnalité au petit comptegouttes et conçoivent l'école confessionnelle tout à l'opposé de l'enseignement de leurs évêques et des pontifes romains. Tant et si bien qu'à lire certains articles de pourfendeurs et à observer quelques-uns de leurs comportements, on dirait les catholiques canadiens-français pris de la rage de l'insecte qui se mange les pattes. Il n'en reste pas moins, rappellerons-nous aux commissaires, qu'à cette question de confessionnalité se lient le grave principe de la liberté religieuse et la formation d'une espèce d'homme qui est l'homme chrétien. Et il reste aussi qu'empêcher de jeunes baptisés de réaliser pleinement leur magnifique personnalité dans la grâce du Christ, ou leur rendre inutilement la tâche plus difficile ou plus aléatoire, cela s'appelle, en bonne langue, sur des jeunes âmes, un viol irrémissible. 
Pourquoi aussi ce chambardement des vieilles humanités, ou du moins ce mépris trop affirmé ? Pourquoi acculer les Collèges classiques à une sorte de suicide ? C'était, paraît-il, la féodalité qu'il fallait abattre. Singuliers féodaux qui, de mon temps, enseignaient pour 40,100 dollars par année, à seule fin de permettre aux fils des pauvres de se faire instruire. Et qui, de cette époque, ne se rappelle que si nos parents avaient dû débourser 200 dollars au lieu des 100 dollars traditionnels, beaucoup de jeunes gens de ma génération, et j'étais de ceux-là, n'auraient pu se faire instruire ? En 1965 l'on ne pouvait accuser ces collèges de stagnation, d'immobilisme. Depuis dix ans leur équipement (bibliothèques, laboratoires), leur personnel enseignant s'étaient renouvelés. Rares les collèges, si même il s'en trouvait, qui ne possédaient leurs spécialistes pour l'enseignement des langues anciennes, de la littérature française, de la philosophie, voire des sciences. Une école d'enseignement secondaire et une Ecole Normale supérieure les pourvoyaient de professeurs compétents. Leur clientèle d'élèves était passée de 20,000 à 46,000 . Les professeurs laïques y entraient en proportion de plus en plus importante. Beaucoup de ces institutions avaient établi le système des sections qui allaient permettre au moins quatre options: humanités gréco-latines, scientifiques, modernes et artistiques. Sans doute ces humanités ont le tort de prendre origine à la Renaissance. Suffit-il de remonter haut dans l'histoire, pour perdre, en route, toute valeur ? Ces générations d'humanistes, issus de la Renaissance, en dépit de ce qu'ils ont pu traîner d'erreurs, ont pourtant fait apparaître une certaine espèce d'hommes. On les aperçoit à l'aurore des grandes découvertes océaniques et à la première compénétration des continents. Ils ont fait du petit cap de l'Europe occidentale le flambeau d'une civilisation assez difficilement égalable. Et ce sont ces mêmes générations qui, bonds par bonds, nous ont amenés à l'âge technique et nucléaire. Que nous promet d'assuré ce que l'on appelle l' "Ecole nouvelle" ? L'autre jour, à la toute récente réunion de l'ACFAS, un professeur de l'un de nos collèges dénonçait ce qu'il appelait la "réaction excessive du Rapport Parent contre le statut privilégié des humanités gréco-latines du Québec". Ce même professeur soutenait, statistiques en mains, qu'à ces humanités plus large part est faite dans la province voisine, l'Ontario. Et il ajoutait cette opinion nullement négligeable pour un peuple qui est à la recherche de sa langue: "L'étude du grec et du latin demeure indispensable à une spécialisation poussée en français." Et je me suis rappelé, à ce propos, quelques petites vérités qu'il m'arrivait, il y a vingt ans, de 
rappeler dans une conférence: Professionnels et culture classique. J'y citais, par exemple, l'opinion d'un monsieur R. M. Hutchison, chancelier de l'Université de Chicago, qui écrivait en 1946: "Le système d'éducation qui tend actuellement à former d'habiles spécialistes sans les pourvoir d'une culture libérale, constitue une sérieuse menace pour: la démocratie." Et je citais aussi cette autre opinion de M. C. R. Young, doyen de la Faculté de génie de l'Université de Toronto, affirmant, pour sa part, qu'en raison de l'avancement des sciences, le futur ingénieur doit posséder "une suffisante culture générale". Mes souvenirs me rappellent aussi les volumes: The Humanities in Canada, de Watson Kirconnell et de A. S. P. Woodhouse (1947), où le Québec n'avait pas à se plaindre de la part louable qui lui était faite. Du même coup je soutenais, quant à moi, qu' "une spécialisation hâtive tend à une diminution ou à un rétrécissement de l'esprit, pour cette raison très simple qu'elle tourne l'esprit vers un champ limité de connaissances et qu'elle se borne par conséquent à l'exercice d'un nombre restreint de facultés", tandis que la supériorité de la culture générale, le mot le dit assez, "procède de son aptitude à développer l'esprit, non sur quelques points, mais en toute sa superficie et profondeur, par un exercice ordonné de toutes les facultés de l'homme". Puis, entre la vieille culture humaniste et la culture moderne, cette différence notable existe qu'en la première l'homme se forme principalement par les lettres, c'est-à-dire par les plus hautes expressions de la pensée humaine, tandis que, dans l'autre, l'objet de l'esprit se compose purement du cosmos en ses multiples matérialités. Sans doute peut-on parler d'humanisme scientifique et même d'humanisme technique, mais dans la mesure où l'homme leur confère de son surplus d'humanité qui lui vient d'autres sources. Aujourd'hui je reprendrais ces mêmes idées pour soutenir qu'en les terribles conjonctures où il s'engage, il faut au Canada français, non principalement la légion de demi ou quart de savants que vont lui former les Instituts, mais, au premier chef, des esprits créateurs, esprits qui se définissent par la vigueur, l'envergure de l'intelligence et de la culture, vigueur et envergure qui se complètent par cette pointe aiguë de l'esprit qu'on appelle la finesse, capable de foncer dans l'inconnu pour lui arracher ses secrets. Esprit de géométrie et de finesse, dirait Pascal. Je veux bien que le Rapport Parent parle beaucoup de méthodes à développer chez l'étudiant, pour l'habiliter à la "créativité". L'important serait de savoir si son court passage, à flots pressés, dans les Instituts et par les mains de quantité de professeurs dont pas un seul peut-être n'aura qualité de maître, lui en four- 
niront les moyens. En Angleterre, dans sa réforme de l'enseignement, le parti travailliste n'a pas osé supprimer les Public Schools, pensionnats d'enseignement secondaires pour garçons ou pour filles, autrement bourgeois et panachés que nos collèges et qui groupent quelque chose comme 45,000 élèves. Plutôt que de subir une réforme dont elle ne veut pas, la plus célèbre de toutes, Eton, serait prête à émigrer en Irlande.

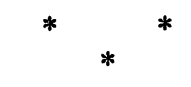

Ces réserves faites, nous ne contestons pas, on l'a vu, les parties valables du Rapport Parent et, de ces parties, nous ne diminuons pas l'importance, pas plus que nous paraît urgente et nécessaire une réforme de notre enseignement et de notre éducation. Qu'a-t-il donc manqué et que manque-t-il encore pour que le peuple s'associe carrément à la réforme qu'on vient de lui offrir ? Il est faux, ainsi que l'écrivent certains, que le peuple se résigne à l'ignorance. Le peuple de toute classe et même une bonne part d'écoliers se sentent inquiets. Il manque à l'un comme aux autres, d'être plus assurés de la valeur magique ou simplement réelle de la nouvelle école. Combien d'instituteurs me l'ont dit, les écoliers s'y sentent un peu perdus; cette impression les tient par trop de servir de cobayes à des expérimentateurs improvisés. Les auteurs du Rapport et ses metteurs en œuvre, il y a lieu de poser la question, se sont-ils rendus compte de l'exceptionnel effort qu'ils demandaient au peuple ? Effort financier d'abord, et cela sous-entend les réactions de contribuables quil soudain voyaient leurs taxes scolaires doublées et parfois même triplées, alors qu'en même temps on leur rebattait les oreilles avec les promesses de la gratuité générale de l'enseignement. Qu'à l'automne de 1966, 50\% des taxes scolaires soient encore impayées est le signe de quelque chose. Les auteurs du Rapport ne se sont pas fait illusion sur la question financière; ils ont écrit ( $I: 78)$, à propos de la montée en flèche du coût de l'enseignement: "Il faut convaincre chacun que les dépenses d'éducation sont un investissement économique et social." Trop d'esprits, et en ce cas je ne parle point seulement du petit peuple, s'interrogeaient en même temps, sur la philosophie, sur les intentions dont s'animait cette nouveauté. J'entends encore un esprit ouvert et grave tel que M. Yves Prévost, habitué à se mettre au-dessus de toute politique, posant cette question, il n'y a pas si longtemps, à ce même propos, devant un auditoire montréalais: "Où allons-nous ? Personne ne le sait ni ne peut le dire." Que ne s'est-on fait la réflexion "qu'une politique ne vaut 
rien si elle n'est pas une doctrine appliquée au réel dans la mesure du possible" ? Chacun a pu avoir devant les yeux deux textes. L'un disait - c'était le Rapport:

Tout en prenant conscience des problèmes qui lui sont particuliers à cause de ses traditions et de son histoire, elle [la province de Québec] doit chercher avec lucidité et sens pratique à doter son système scolaire de structures adaptées à ses besoins. La conception moderne de l'éducation vise à préparer chaque citoyen à gagner sa vie par un travail utile et à assumer intelligemment ses responsabilités sociales. (I: 83-84).

Un autre texte venu d'une autre source affirmait:

Un système d'éducation doit s'inspirer des valeurs propres de la civilisation québecoise qui ne sont pas seulement celles qui tendent à la langue et à la culture, mais aussi les valeurs chrétiennes, celles-ci ayant contribué autant que celles-là à forger l'âme de notre peuple.

Le rapprochement de ces deux textes permet de saisir les tendances pragmatistes du système d'enseignement du Rapport et d'en soupçonner les origines. Au surplus, tant des nouveaux docteurs de la nouvelle et vague philosophie scolaire avouaient leur dessein de faire du jeune Canadien français un nord-américain. D'un mot: à mesure que le gouvernement du Québec se nationalise, il a trop paru que son système d'enseignement se dénationalise. A coup sûr la mise en ration des vieilles humanités et la destruction de l'ancien système d'enseignement pouvaient éveiller les mêmes inquiétudes. Je repose alors une autre question: dans nos milieux officiels de l'enseignement, a-t-on bien aperçu, en cette perspective, le grave aspect que pouvait prendre l'Ecole nouvelle? Jusqu'ici les vieilles civilisations méditerranéennes étaient restées l'axe vivant de la culture et de l'esprit canadien-français. Soudair l'on invitait les Québecois à changer l'axe de leur culture natale pour l'aligner en somme sur la civilisation anglo-américaine. En d'autres termes on proposait à un peuple un geste exceptionnel en histoire: un retournement d'âme, et la plus grave et la plus profonde des révolutions: celle de l'esprit. Parmi ceux qui réfléchissent, et de ces esprits, il en est plus qu'on ne pense, que ne pouvait signifier cette américanisation voulue ou non de l'enseignement ? L'on a déjà nourri et l'on nourrit encore l'illusion de sauver, au Québec, la vieille langue française, en dépit de l'économie anglo-américaine 
où l'ouvrier gagne sa vie en anglais. L'illusion devient gigantesque, s'il faut, par surcroît, américaniser l'enseignement québecois, à moins que, pour survivre comme nation distincte, la formule par excellence soit devenue de ressembler le plus possible à son grand voisin.

C'étaient là les obstacles, l'état d'esprit qu'il fallait affronter. Les obstacles, comment les écarter ? Les inquiétudes, comment les apaiser? Et pour entraîner le peuple au vaste effort qu'on exigeait de lui, qui possédait assez de panache pour en imposer ? Et quelle mystique agissante eût déterminé ce qui est au départ de tous les grands mouvements collectifs? Et cette mystique, de quoi l'eût-on composée ?

On trouve à critiquer aujourd'hui ceux qui ont pris la relève en cette réforme scolaire. On leur fait grief de paraître piétiner. Plaignons-les plutôt d'avoir à repenser un problème de cette taille. S'ils osent un redressement - l'oseront-ils ? - le redressement, on l'a vu, ne pourra s'affirmer que vigoureux et considérable. Il est plus facile de défaire que refaire. Souhaitons néanmoins que tout en sauvegardant les parties valables du Rapport, parties d'importance, on parvienne à harmoniser l'ancien et le nouveau système dans une cohérence qui n'abdique rien du progrès. Le projet s'impose d'une grande réforme scolaire et d'y engager à fond l'élan et la volonté ardente de la nation. Mais cet élan ne saurait se passer, comme on dit aujourd'hui, d'une "motivation" à sa mesure, ou de ce que l'on appelle encore plus savamment "la démocratie de participation".

LIONEL GROULX, ptre 